Quim. Nova, Vol. 36, No. 3, 468-473, 2013

\title{
EXTRAÇÃO DE BIFENILAS POLICLORADAS DE AMOSTRAS DE LEITE MATERNO: OTIMIZAÇÃO UNIVARIADA VERSUS PLANEJAMENTO EXPERIMENTAL
}

\author{
Cláudia Hoffmann Kowalski* e Fabio Augusto \\ Instituto de Química, Universidade Estadual de Campinas, CP 6154, 13084-971 Campinas - SP, Brasil \\ Helena Teixeira Godoy \\ Faculdade de Engenharia de Alimentos, Universidade Estadual de Campinas, CP 6121, 13083-862 Campinas - SP, Brasil
}

Recebido em 1/6/12; aceito em 10/9/12; publicado na web em 6/2/13

\begin{abstract}
EXTRACTION OF POLYCHLORINATED BIPHENYLS IN BREAST MILK SAMPLES: UNIVARIATED OPTIMIZATION VERSUS EXPERIMENTAL DESIGN. The convenience of the multivariate optimization of SPME procedures through ANOVA calculated using Doehlert designs has been demonstrated for twelve PCBs in the complex matrix of milk. For this study, the main parameters of the extraction were selected and valued through univariate and multivariate optimization. In addition, the analysis of variance allowed identification of the statistically significant variables in this model: high temperature $\left(95^{\circ} \mathrm{C}\right)$ and ionic strength $(36 \%$ $\mathrm{m} / \mathrm{v}$ ) proved significant for all the PCBs while intermediate time $(70 \mathrm{~min})$ and low methanol concentration (5\% v/v) also contributed to the extraction of the majority of these PCBs.
\end{abstract}

Keywords: PCBs; SPME; Doehlert design.

\section{INTRODUÇÃO}

As bifenilas policloradas (PCBs) constituem um grupo de contaminantes altamente tóxicos classificados como poluentes orgânicos persistentes (POPs). Tal atribuição deve-se a três características básicas: persistência ambiental, bioacumulação e alta toxicidade. ${ }^{1}$ A persistência desses compostos ocorre em função de sua baixa degradação por processos bióticos e abióticos, acarretando em elevada meia-vida no ambiente, que pode chegar a anos ou décadas. ${ }^{2}$

Os PCBs são absorvidos pelos organismos através da alimentação, respiração e pele. Alimentos como leite e derivados, carne e peixe têm sido identificados como a principal rota de entrada dos PCBs e pesticidas nos organismos humanos. ${ }^{3-5}$ Essas substâncias, depois de absorvidas, são distribuídas através da corrente sanguínea para os vários tecidos e órgãos do corpo, sendo armazenadas principalmente nos tecidos ricos em gordura. No caso das mulheres, ocorre um grande acúmulo desses compostos no leite e, consequentemente, nos recém-nascidos por elas amamentados. ${ }^{6} \mathrm{~A}$ contaminação do leite materno por PCBs é de grande impacto, pois é o primeiro alimento do recém-nascido, constituindo um grande problema para sua saúde. Um estudo publicado recentemente no Brasil mostrou que a contaminação do leite materno com PCBs está altamente relacionada com o grau de industrialização da região, sendo que na quatro capitais brasileiras estudadas (São Paulo, Vitória, Florianópolis e Rio Branco) as porcentagem de amostras contaminadas foram de 100, 80, 60 e $0 \%$, respectivamente. ${ }^{7}$

Dentre as técnicas de extração de contaminantes mais utilizadas na atualidade, a microextração em fase sólida (SPME) é bastante pertinente para os casos onde se dispõe de pequenas quantidades de amostra, como é o caso dos fluídos biológicos. De forma a maximizar a eficiência de extração dos compostos de interesse, vários parâmetros extrativos podem ser otimizados como o tipo de fibra, a concentração e o volume da amostra, a temperatura e o tempo de extração e a adição de reagentes e solutos.

Duas abordagens podem ser empregadas para realizar essa otimização: univariada e multivariada. Na primeira, cada variável é estudada separadamente, enquanto que na segunda, as variáveis são

*e-mail: claukowalski@gmail.com estudadas simultaneamente, tornando possível distinguir interações entre elas que não seriam detectadas na abordagem tradicional. ${ }^{8-10}$

Uma abordagem muito conveniente de otimização multivariada foi proposta por Doehlert, em 1970, ${ }^{11}$ pois permite o estudo de um número grande de variáveis utilizando um número reduzido de experimentos, tornando-o mais eficiente quando comparado a outros planejamentos..$^{10,12-14}$ Outra vantagem deste planejamento é que o número de níveis não é o mesmo para todas as variáveis. Essa propriedade permite a livre escolha de quais fatores serão avaliados em um número maior ou menor de níveis, conforme sua importância no sistema ou de forma a obter maiores informações sobre o comportamento de uma ou outra variável naquele sistema. ${ }^{9}{ }^{15}$ Essas características o tornam muito útil na otimização de experimentos nas mais variadas áreas da química analítica, ${ }^{16}$ considerando diferentes técnicas instrumentais de preparo e análise bem como diferentes tipos de amostras e analitos. ${ }^{7,16-20}$

Neste trabalho foi proposta a otimização das condições experimentais para a extração de PCBs de amostras de leite materno utilizando a técnica de microextração em fase sólida no modo headspace (HS-SPME). Para isso, utilizou-se tanto a abordagem univariada como a multivariada, sendo possível comparar os resultados obtidos em cada estudo e identificar as vantagens e desvantagens de cada tratamento. Além disso, a qualidade do ajuste dos modelos individuais obtidos de cada resposta foi estimada através da análise de variância (ANOVA), possibilitando identificar quais as variáveis que influenciam na extração dos PCBs bem como os melhores valores experimentais para cada uma delas.

\section{PARTE EXPERIMENTAL}

\section{Reagentes e materiais}

As soluções padrão de bifenilas policloradas foram adquiridas da AccuStandard (New Haven, CT), com concentração de 3,5 $\mu \mathrm{g} \mathrm{mL} \mathrm{mL}^{-1}$ de cada PCB. Os isômeros estudados neste trabalho foram o PCB 28 (2,4,4'-triclorobifenil), PCB 52 (2,2',5,5'-tetraclorobifenil), PCB 74 (2,4,4`,5-tetraclorobifenil), PCB 101 (2,2`,4,5,5'-pentaclorobifenil),

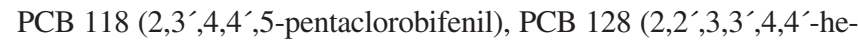
xaclorobifenil), PCB 138 (2,2’,3,4,4’,5-hexaclorobifenil), PCB 153 
(2,2',4,4',5,5'-hexaclorobifenil), PCB 156 (2,3,3’,4,4',5-hexaclorobifenil), PCB 170 (2,2, $3,3^{\prime}, 4,4^{\prime}, 5$-heptaclorobifenil), PCB 180

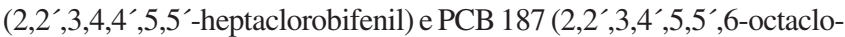
robifenil). Metanol grau HPLC, tolueno grau HPLC e NaOH P.A. foram obtidos da Merck (Darmstadt, Alemanha), o NaCl P.A. da Ecibra (São Paulo, Brasil) e o iso-octano grau HPLC da Mallinckrodt (Kentucky, PA). Água deionizada purificada através de um sistema Millipore Milli-Q (Bedford, MA) também foi utilizada. As fibras utilizadas foram a de polidimetilsiloxano (PDMS) com espessura de recobrimento de $100 \mu \mathrm{m}$ e de polidimetilsiloxano-divinilbenzeno (PDMS-DVB) com espessura de $65 \mu \mathrm{m}$, adaptadas em um aplicador apropriado, sendo esse conjunto fornecido pela Supelco (Bellefonte, PA).

\section{Amostras}

Vinte amostras de leite materno foram obtidas de um banco de leite da cidade de São Paulo, sendo mantidas congeladas da coleta até o preparo das amostras. Para os estudos de otimização, as amostras foram descongeladas e misturadas de forma a obter um pool com volume suficiente para todos os testes. Esse pool foi contaminado com uma solução de trabalho contendo todos os PCBs, obtendo-se amostras contaminadas com 9,25 $\mu \mathrm{g} \mathrm{L}^{-1}$ de cada PCB. Elas foram mantidas de 2 a $4{ }^{\circ} \mathrm{C}$ por $24 \mathrm{~h}$ para permitir o equilíbrio entre os compostos biológicos ativos do leite e os compostos organoclorados adicionados. ${ }^{21}$ Após esse período, realizou-se a extração dos compostos.

\section{Instrumentação}

As análises cromatográficas foram realizadas em um cromatógrafo AutoSystemXL GC-ECD (Perkin-Elmer, Norwalk, CT) com uma coluna HP-1MS (100\% polidietilsiloxano) $30 \mathrm{~m} \mathrm{x} \mathrm{0,32} \mathrm{mm} \mathrm{x}$ $0,25 \mu \mathrm{m}$. O injetor foi operado no modo splitless. A programação de temperatura do forno foi: $40^{\circ} \mathrm{C}$ por $2 \mathrm{~min}$, rampa de $30^{\circ} \mathrm{C} \mathrm{min}{ }^{-1}$ até $190^{\circ} \mathrm{C}$, mantido por $5 \mathrm{~min}$, rampa de $5^{\circ} \mathrm{C} \mathrm{min}^{-1}$ até $220^{\circ} \mathrm{C}$, mantido por $5 \mathrm{~min}$, rampa de $20^{\circ} \mathrm{C} \mathrm{m^{-1 }}$ até $300^{\circ} \mathrm{C}$, mantido por $1 \mathrm{~min}$. As temperaturas do injetor e detector foram 280 e $320^{\circ} \mathrm{C}$, respectivamente. O gás de arraste foi hélio com pureza de $99,999 \%$ (White Martins) e vazão de $1,3 \mathrm{~mL} \mathrm{~min}{ }^{-1}$. O gás makeup utilizado foi o nitrogênio com pureza de 99,999\% (White Martins).

\section{Otimização univariada}

Foram estudados o efeito da presença da matriz (água e leite), o tipo de recobrimento da fibra (PDMS $100 \mu \mathrm{m}$ e PDMS-DVB), o tempo de extração (20 a $90 \mathrm{~min}$ ), a temperatura de extração (de 27 até $\left.80{ }^{\circ} \mathrm{C}\right)$, a adição de $\mathrm{NaCl}(0$ a $36 \%$ ), a adição de reagente (metanol e tolueno) e a saponificação da gordura com $\mathrm{NaOH}$ (0 e 20\%). Para todos os experimentos, foram colocadas alíquotas de $5 \mathrm{~mL}$ de amostra em frascos e termostatizados em banho de água (ColeParmer - Vernon Hills, IL) sob agitação magnética (1200 rpm) por 10 min para o equilíbrio amostra/headspace. Então, a fibra de SPME foi exposta ao headspace da amostra por tempo pré-definido, sendo posteriormente recolhida e exposta ao injetor do cromatógrafo a 280 ${ }^{\circ} \mathrm{C}$ por 5 min. Os resultados obtidos foram transferidos e tratados no programa Origin 5.0 (Microcal Software Inc., Northampton, MA).

\section{Planejamento experimental}

Foi utilizada somente a fibra de PDMS $100 \mu \mathrm{m}$, e o procedimento genérico foi similar ao descrito no item anterior. A adição de $\mathrm{NaCl}$ (para ajuste da força iônica) $\left(\mathrm{C}_{\mathrm{NaCl}}\right)$, a temperatura de extração $(\mathrm{T})$, o tempo de extração $(\mathrm{t})$ e a adição do cossolvente metanol $\left(\mathrm{C}_{\mathrm{MeOH}}\right)$ foram os quatro parâmetros da HS-SPME otimizados. As faixas de trabalho,
Tabela 1. Condições operacionais segundo distribuição de Doehlert para as quatro variáveis HS-SPME estudadas

\begin{tabular}{ccccc}
\hline $\begin{array}{c}\text { Ensaio } \\
\text { experimental }\end{array}$ & $\begin{array}{c}\mathrm{C}_{\mathrm{NaCl}}(\% \mathrm{~m} / \mathrm{v}) \\
5 \text { níveis }\end{array}$ & $\begin{array}{c}\mathrm{T}\left({ }^{\circ} \mathrm{C}\right) \\
7 \text { níveis }\end{array}$ & $\begin{array}{c}\mathrm{t}(\mathrm{min}) \\
7 \text { níveis }\end{array}$ & $\begin{array}{c}\mathrm{C}_{\mathrm{MeOH}}(\% \mathrm{v} / \mathrm{v}) \\
3 \text { níveis }\end{array}$ \\
\hline $1^{\mathrm{a}}$ & $0(18)^{\mathrm{b}}$ & $0(70)$ & $0(70)$ & $0(5)$ \\
2 & $1(36)$ & $0(70)$ & $0(70)$ & $0(5)$ \\
3 & $0,5(27)$ & $0,866(95)$ & $0(70)$ & $0(5)$ \\
4 & $0,5(27)$ & $0,289(78,3)$ & $0,817(100)$ & $0(5)$ \\
5 & $0,5(27)$ & $0,289(78,3)$ & $0,204(77,5)$ & $0,791(10)$ \\
6 & $-1(0)$ & $0(70)$ & $0(70)$ & $0(5)$ \\
7 & $-0,5(9)$ & $-0,866(45)$ & $0(70)$ & $0(5)$ \\
8 & $-0,5(9)$ & $-0,289(61,7)$ & $-0,817(40)$ & $0(5)$ \\
9 & $-0,5(9)$ & $-0,289(61,7)$ & $-0,204(62,5)$ & $-0,791(0)$ \\
10 & $0,5(27)$ & $-0,866(45)$ & $0(70)$ & $0(5)$ \\
11 & $0,5(27)$ & $-0,289(61,7)$ & $-0,817(40)$ & $0(5)$ \\
12 & $0,5(27)$ & $-0,289(61,7)$ & $-0,204(62,5)$ & $-0,791(0)$ \\
13 & $-0,5(9)$ & $0,866(95)$ & $0(70)$ & $0(5)$ \\
14 & $0(0)$ & $0,577(86,7)$ & $-0,817(40)$ & $0(5)$ \\
15 & $0(0)$ & $0,577(86,7)$ & $-0,204(62,5)$ & $-0,791(0)$ \\
16 & $-0,5(9)$ & $0,289(78,3)$ & $0,817(100)$ & $0(5)$ \\
17 & $0(18)$ & $-0,577(53,3)$ & $0,817(100)$ & $0(5)$ \\
18 & $0(18)$ & $0(70)$ & $0,613(92,5)$ & $-0,791(0)$ \\
19 & $-0,5(9)$ & $0,289(78,3)$ & $0,204(77,5)$ & $0,791(10)$ \\
20 & $0(18)$ & $-0,577(53,3)$ & $0,204(77,5)$ & $0,791(10)$ \\
21 & $0(18)$ & $0(70)$ & $-0,613(47,5)$ & $0,791(10)$ \\
\hline
\end{tabular}

${ }^{a}$ Ponto central com três repetições. ${ }^{b}$ Fora do parêntese: valor codificado; dentro do parêntese: valor real.

calculadas de acordo com a Equação 1, estão demonstradas na Tabela 1.

$$
C_{i}=\left\{\frac{X_{i}-X_{i}^{0}}{\Delta X_{i}}\right\} \alpha
$$

onde $C i$ é o valor codificado para o nível do fator $i, X i$ é o valor experimental, $X_{i}^{0}$ é o valor experimental no ponto central, $\Delta X i$ é a distância entre o valor experimental no ponto central e o experimental no nível superior ou inferior e $\alpha$ é o maior valor limite codificado na matriz para cada fator.

A regressão linear múltipla (MLR) foi utilizada para encontrar um modelo matemático (Equação 2) apropriado para cada PCB estudado, a fim de descrever o comportamento de cada composto em função das variáveis experimentais de extração avaliadas.

$$
\begin{aligned}
\mathrm{z}= & b_{0}+b_{1} \mathrm{C}_{\mathrm{NaCl}}+b_{2} \mathrm{~T}+b_{3} \mathrm{t}+b_{4} \mathrm{C}_{\mathrm{MeOH}}+b_{11} \mathrm{C}_{\mathrm{NaCl}}^{2}+b_{22} \mathrm{~T}^{2} \\
& +b_{33} \mathrm{t}^{2}+b_{44} \mathrm{C}_{\mathrm{MeOH}}^{2}+b_{12} \mathrm{C}_{\mathrm{NaCl}} \mathrm{T}+b_{13} \mathrm{C}_{\mathrm{NaCl}} \mathrm{t}+b_{14} \mathrm{C}_{\mathrm{NaCl}} \mathrm{C}_{\mathrm{MeOH}} \\
& +b_{23} \mathrm{~T} \mathrm{t}+b_{24} \mathrm{TC}_{\mathrm{MeOH}}+b_{34} \mathrm{C}_{\mathrm{MeOH}}
\end{aligned}
$$

onde $z$ é área de pico do PCB e $b$ corresponde aos coeficientes da regressão.

Cada PCB apresentará uma equação de regressão com seus respectivos coeficientes, ao nível de significância de 0,05 .

A qualidade dos modelos individuais foi estimada através da análise de variância (ANOVA). Os cálculos dos modelos de regressão e das análises de variância foram feitos através de planilhas eletrônicas elaboradas no software Excel $2003^{15}$ e no Statistica 6.0 (StatSoft Inc, Tulsa, OK).

\section{RESULTADOS E DISCUSSÃO}

\section{Otimização univariada}

Os parâmetros estudados experimentalmente foram os seguintes: 


\section{Efeito da matriz}

A Figura 1 mostra as áreas dos picos dos PCBs após a contaminação de uma amostra de água deionizada e de leite com PCBs. Conforme esperado, a influência da matriz foi grande, observando-se uma redução de aproximadamente $90 \%$ na área dos picos.

\section{Tipo de recobrimento da fibra}

Apesar da eficiência de extração ser maior com a fibra de PDMSDVB, observou-se maior variação entre as áreas obtidas nas replicatas (Figura 2). Além disso, a corrida de um branco entre as amostras mostrou que essa fibra apresenta efeito de memória significativo, quando comparada à PDMS. Esses resultados estão de acordo com

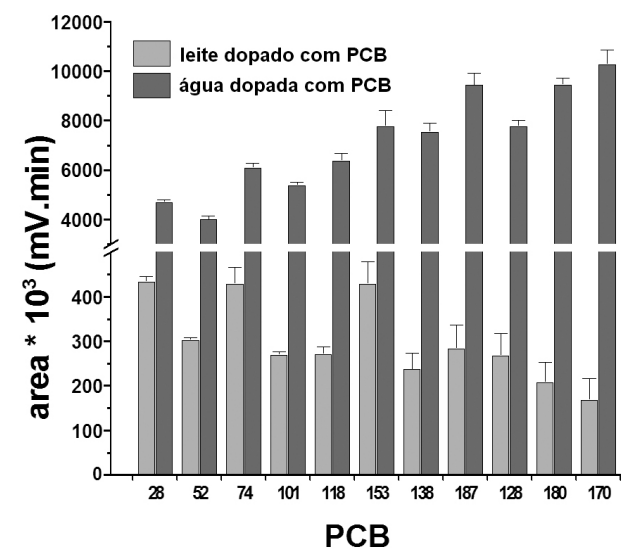

Figura 1. Avaliação do efeito de matriz na extração de PCB por HS-SPME-GC-ECD. Água e leite materno contaminados com 9,25 $\mu \mathrm{g} L^{-1}$ de cada PCB. $C_{\mathrm{NaCl}}=360 \mathrm{~g} \mathrm{~L}^{-1} ; \mathrm{T}=85^{\circ} \mathrm{C} ; t=60 \mathrm{~min}$
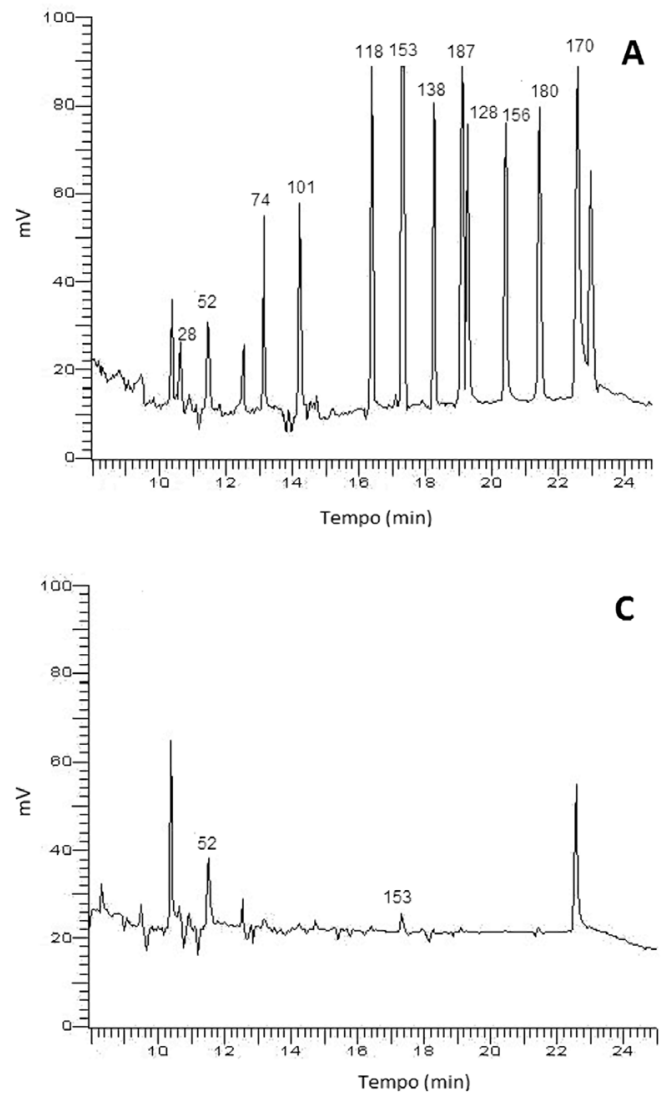

os obtidos por Montes et al., ${ }^{22}$ que calcularam o carryover para ambas as fibras para os congêneres de PCB com maior cloração, e encontraram 2 a $13 \%$ e 15 a 35\% para a fibra PDMS e PDMS-DVB, respectivamente. A partir de então, todos os experimentos foram conduzidos com fibras de PDMS $100 \mu \mathrm{m}$.

\section{Tempo de extração}

Para os PCB de menor peso molecular (PCB 28, 52, 74, 101 e 118), 50 min foi suficiente para atingir o equilíbrio. Já para os PCB com maior cloração (PCB 180 e 170), mesmo após 90 min o equilíbrio não foi alcançado conforme observado na Figura 1S. Entretanto, para análises de rotina, 90 min ou mais de extração seria

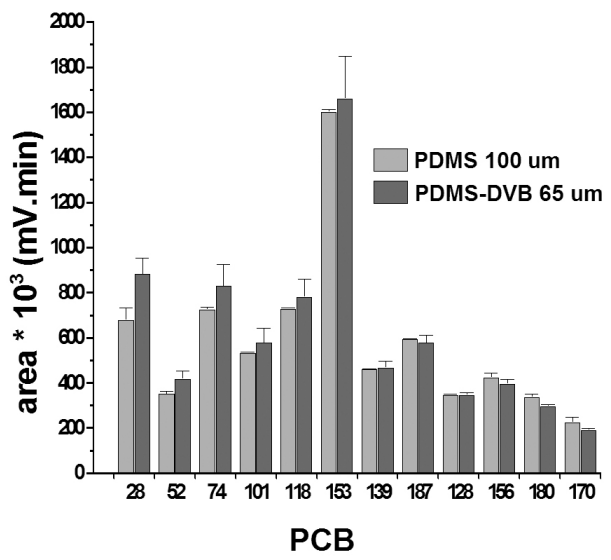

Figura 2. Perfis de extração dos PCB para as fibras PDMS 100 нm e PDMS-DVB $65 \mu \mathrm{m}$. Leite materno contaminado com 9,25 $\mu \mathrm{g} L^{-1}$ de cada PCB. $C_{\mathrm{NaCl}}$ $=360 \mathrm{~g} \mathrm{~L}{ }^{-1} ; T=85^{\circ} \mathrm{C} ; t=60 \mathrm{~min}$
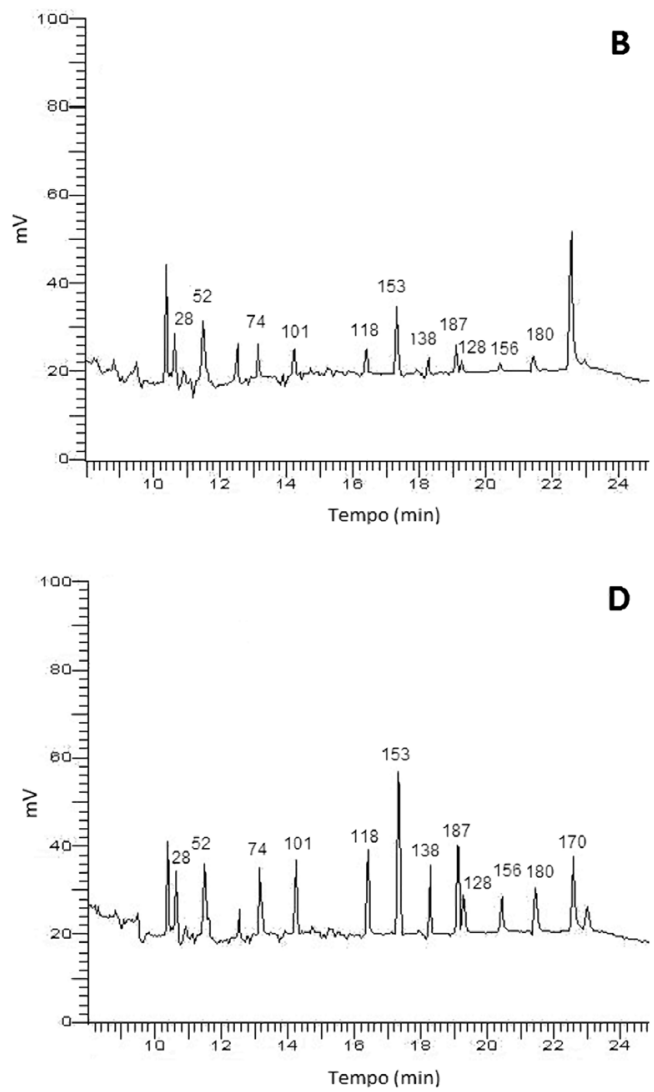

Figura 3. Cromatogramas obtidos no planejamento experimental. (A) $C_{\mathrm{NaCl}}: 27 \%$, T: $95{ }^{\circ} \mathrm{C}$, t: 70 min e $C_{\mathrm{MeOH}}: 5 \%$; (B) $C_{\mathrm{NaCl}}: 9 \%$, T: $45{ }^{\circ} \mathrm{C}$, $t: 70 \mathrm{~min}$ e $C_{\mathrm{MeOH}}$ : $5 \%$; (C) $C_{\mathrm{NaCl}}: 18 \%, \mathrm{~T}: 70{ }^{\circ} \mathrm{C}$, t: 92,5 min e $C_{\mathrm{MeOH}}: 0 \%$; (D) $C_{\mathrm{NaCl}}: 18 \%, \mathrm{~T}: 70{ }^{\circ} \mathrm{C}, t: 70 \mathrm{~min}$ e $C_{\mathrm{MeOH}}: 5 \%$ 
muito tempo, mostrando a necessidade de otimizar outros parâmetros que possibilitem ao sistema alcançar o equilíbrio com menos tempo.

\section{Temperatura de extração}

A Figura 2S, material suplementar, mostra a grande influência deste parâmetro na eficiência de extração. $\mathrm{O}$ aumento da temperatura durante a extração aumenta a difusão do analito ao redor da fibra. Contudo, no modo de extração utilizando o headspace da amostra, a temperatura ajuda na transferência dos analitos da matriz da amostra para o seu headspace. ${ }^{23}$ Esse fenômeno é observado em todos os PCBs analisados e existe uma correlação direta entre o peso molecular e a temperatura necessária para que o equilíbrio entre as fases (amostra/ headspace/fibra) seja obtido. Por isso, verifica-se a necessidade de testar temperaturas de extração ainda mais altas para os PCBs de maior peso molecular.

\section{Adição de $\mathrm{NaCl}$}

Na Figura 3S, material suplementar, é possível verificar o aumento da quantidade de PCBs extraída quando da adição de $0,9 \mathrm{~g}$ de $\mathrm{NaCl}$ em $5 \mathrm{~mL}$ da matriz, principalmente para os PCBs de maior peso molecular. A presença de eletrólitos fortes, como $\mathrm{NaCl}$, aumenta a atividade dos analitos, levando à redução na solubilidade dos mesmos, que são mais facilmente retidos pela fibra, deslocando o equilíbrio de extração. Mas, segundo Penalver, ${ }^{23}$ esse processo não é válido em todos os casos, sendo que esse efeito de aumento da extração depende da polaridade do analito, da concentração do sal e da matriz na qual o analito se encontra.

\section{Adição de um ou mais cossolventes}

Na mesma Figura 3S, material suplementar, vê-se que o tolueno (baixa polaridade) diminuiu drasticamente a extração dos PCBs da matriz; já a adição de metanol (média polaridade) em uma solução saturada de $\mathrm{NaCl}$ proporcionou um ligeiro aumento da extração, quando comparada a uma solução somente saturada. Estudos demonstram que amostras aquosas adicionadas de pequenas quantidades de solventes orgânicos miscíveis em água, tais como metanol ou acetonitrila, podem ter ampliada a eficiência de extração de compostos altamente hidrofóbicos. ${ }^{24}$ Entretanto, esse efeito é devido ao aumento na solubilidade dos analitos no meio. Um aumento excessivo de cossolvente pode mudar as constantes de equilíbrio amostra/headspace e amostra/fibra para os analitos, reduzindo as quantidades extraídas na condição de equilíbrio.

\section{Saponificação da gordura}

Segundo Llompart, ${ }^{25}$ a saponificação da gordura do leite ajuda na transferência dos PCBs da matriz para a fibra, além de atuar como um clean up e, assim, aumentar a seletividade e confiança na identificação dos picos cromatográficos. Isso ocorre porque a saponificação da gordura em seus correspondentes gliceróis e ácidos graxos facilita a liberação dos PCBs da matriz. Esse procedimento pode ser de grande importância principalmente para amostras com grandes quantidades de gordura (leite maduro), nas quais os PCBs estarão mais fortemente ligados. No entanto, diferentemente dos resultados obtidos naquele estudo, ${ }^{25}$ observa-se na Figura $4 \mathrm{~S}$, material suplementar, que com a adição de $20 \%$ de $\mathrm{NaOH}$ na amostra a área extraída foi muito pequena. Além disso, a presença de $\mathrm{NaOH}$ suprimiu o efeito da força iônica exercida pela adição de $\mathrm{NaCl}$. Ambos os resultados podem ser comparados aos obtidos quando a extração foi feita sem $\mathrm{NaOH}$ e $\mathrm{NaCl}$. Sendo assim, essa variável foi descartada do planejamento experimental.

\section{Planejamento Doehlert}

Os cromatogramas da Figura 3 mostram quatro condições distintas obtidas do planejamento: a maior soma de áreas (A), a menor soma de áreas (B), o ponto central do experimento (C) e a condição mais uniforme (D). De forma geral, o cromatograma A dessa figura apresentou áreas cromatográficas bastante expressivas, principalmente para os PCBs de maior peso molecular. Comparando o cromatograma A (extração a $95^{\circ} \mathrm{C}$ ) com os demais cromatogramas (extração a 45 , 70 e $70^{\circ} \mathrm{C}$, respectivamente), observa-se uma correlação direta entre a temperatura e a eficiência da extração. $\mathrm{O}$ mesmo pode ser observado para a adição de $\mathrm{NaCl}$, já que a solução a $27 \%$ (cromatograma $\mathrm{A}$ ) foi bem mais eficiente que as demais (9, 18 e 18\%).

Apesar dessa análise simples e pontual, deve-se considerar que as interações de $2^{\mathrm{a}}$ e $3^{\mathrm{a}}$ ordens são tão importantes quanto a de $1^{\mathrm{a}}$ ordem, necessitando de uma abordagem bem embasada estatisticamente.

A Tabela 2 mostra os coeficientes polinomiais $b$ obtidos para cada PCB, ao nível de significância de 0,05 , definidos de acordo com o modelo quadrático da Equação 1.

Tabela 2. Coeficientes dos modelos polinomiais determinados por MLR para os PCBs individuais

\begin{tabular}{|c|c|c|c|c|c|c|c|c|c|c|c|c|}
\hline \multirow{2}{*}{ Coeficientes } & \multicolumn{12}{|c|}{ PCB \# } \\
\hline & 28 & 52 & 74 & 101 & 118 & 153 & 138 & 187 & 128 & 156 & 180 & 170 \\
\hline$b_{0}$ & 345 & 505 & 161 & 245 & 222 & 643 & 215 & 222 & 97 & 113 & 210 & 76 \\
\hline \multicolumn{13}{|c|}{ Coeficientes de $1^{\mathrm{a}}$ ordem } \\
\hline$b_{1}$ & 117 & 114 & 134 & 206 & 234 & 738 & 240 & 327 & 46 & 143 & 173 & 129 \\
\hline$b_{2}$ & 196 & 248 & 607 & 736 & 1188 & 3338 & 1217 & 1856 & 963 & 1016 & 1220 & 878 \\
\hline$b_{3}$ & 61 & 148 & -3 & -52 & -69 & -139 & $-\underline{70}$ & $-\underline{117}$ & $-\underline{47}$ & $-\underline{46}$ & -37 & $-\underline{45}$ \\
\hline$b_{4}$ & -58 & -19 & $\underline{-133}$ & $-\underline{237}$ & $-\underline{293}$ & $-\underline{619}$ & $-\underline{-255}$ & $-\underline{390}$ & $-\underline{168}$ & $-\underline{200}$ & $-\underline{237}$ & $-\underline{124}$ \\
\hline \multicolumn{13}{|c|}{ Coeficientes de $2^{\mathrm{a}}$ ordem } \\
\hline$b_{11}$ & 76 & 270 & 81 & 159 & 189 & 391 & 133 & 196 & 108 & 119 & 109 & 79 \\
\hline$b_{22}$ & -12 & -60 & 567 & 578 & 1264 & 4106 & 1539 & 2416 & 1349 & 1405 & 1637 & 1349 \\
\hline$b_{33}$ & 190 & 202 & -28 & -8 & -66 & $-\underline{456}$ & $-\underline{129}$ & $-\underline{324}$ & $-\underline{202}$ & $-\underline{227}$ & $-\underline{289}$ & $-\underline{-248}$ \\
\hline$b_{44}$ & 104 & 76 & 81 & 130 & 129 & 58 & 71 & 16 & -18 & -10 & 9 & -81 \\
\hline \multicolumn{13}{|c|}{ Coeficientes de interação } \\
\hline$b_{12}$ & 116 & 363 & 576 & 695 & 980 & 3770 & 1035 & 1643 & 151 & 699 & 933 & 695 \\
\hline$b_{13}$ & -109 & -137 & -297 & $-\underline{377}$ & $\underline{-536}$ & $-\underline{1659}$ & $-\underline{-518}$ & $-\underline{794}$ & $\underline{-151}$ & $-\underline{343}$ & $-\underline{-395}$ & $-\underline{337}$ \\
\hline$b_{14}$ & -164 & -126 & -143 & -184 & $-\underline{236}$ & $-\underline{968}$ & $-\underline{-282}$ & $-\overline{-383}$ & $\overline{-15}$ & $-\underline{165}$ & $-\overline{267}$ & $-\overline{180}$ \\
\hline$b_{23}$ & -436 & -437 & -131 & $-\underline{350}$ & $-\underline{270}$ & -93 & -84 & -50 & 79 & 80 & 151 & 160 \\
\hline$b_{24}$ & -102 & -407 & -69 & $-\underline{336}$ & $-\underline{319}$ & -466 & $-\underline{236}$ & $-\underline{-310}$ & $-\underline{73}$ & $-\underline{146}$ & $-\underline{273}$ & 75 \\
\hline$b_{34}$ & 88 & 87 & -98 & -244 & $-\underline{423}$ & -1580 & -496 & $-\underline{-908}$ & $-\underline{-501}$ & $-\underline{-577}$ & $\underline{-699}$ & $-\underline{555}$ \\
\hline
\end{tabular}

Valores sublinhados indicam valores de Fcal significativos em relação aos valores da distribuição de $\mathrm{F}$ tabelado. 
A observação dos efeitos significativos dos modelos individuais evidenciou a relação não linear entre as áreas cromatográficas obtidas para cada conjunto experimental SPME, devido à presença de vários efeitos significativos de $2^{\mathrm{a}}$ ordem. Além disso, podem-se notar, também, muitos efeitos significativos de interação entre as variáveis. Sendo assim, fica evidente que a otimização univariada não é a melhor opção, já que não considera as interações e por isso pode conduzir à seleção de variáveis operacionais distantes do ótimo.

Verifica-se que, para as variáveis $\mathrm{C}_{\mathrm{NaCl}}$ e $\mathrm{T}$, a maioria dos coeficientes se apresentaram positivos, indicando que a adição de $\mathrm{NaCl}$ e o aumento na temperatura de extração aumentam a eficiência de extração dos PCBs. Do contrário, para as variáveis t e $\mathrm{C}_{\mathrm{MeOH}}$ os coeficientes se mostraram em grande parte negativos, ou seja, períodos intermediários de tempo e baixas concentrações de metanol ampliam a eficiência de extração na região experimental avaliada.

A qualidade do ajuste dos modelos individuais foi estimada através da análise de variância (ANOVA) apresentada na Tabela 3.
De acordo com a Tabela 3, as regressões para todos os PCBs estudados foram estatisticamente válidas, considerando-se $95 \%$ de confiabilidade. Entretanto, ocorreu falta de ajuste dos modelos para a maioria dos analitos ao mesmo nível de confiança. Além disso, pelos coeficientes significativos das interações entre as variáveis ( $p$ menor que o nível de significância $\alpha=0,05$ ) pode-se estimar que o efeito das variações dos parâmetros operacionais na eficiência de extração não é independente.

Na Tabela 4 é apresentada uma comparação entre as melhores condições experimentais do estudo univariado e do planejamento experimental. Apesar de, em ambos os casos, diferentes condições serem sugeridas para diferentes PCBs, as áreas cromatográficas obtidas no planejamento foram maiores e só no planejamento foi possível identificar uma condição experimental com máxima eficiência de extração (cromatograma A) e uma condição bastante uniforme para a extração de todos os PCBs simultaneamente (cromatograma D).

Tabela 3. Análise de variância das regressões quadráticas dos modelos individuais dos PCBs, ao nível de significância de 0,05

\begin{tabular}{|c|c|c|c|c|c|c|c|c|c|c|c|}
\hline $\begin{array}{l}\text { Fonte de } \\
\text { variação }\end{array}$ & $\begin{array}{c}\text { Soma } \\
\text { quadrática } \\
\text { (SQ) }\end{array}$ & $\begin{array}{c}\text { Graus de } \\
\text { liberdade } \\
\text { (GL) }\end{array}$ & $\begin{array}{c}\text { Média } \\
\text { quadrática } \\
\text { (MQ) }\end{array}$ & $\mathrm{F}_{\text {calc }}$ & $p$ & $\begin{array}{l}\text { Fonte de } \\
\text { variação }\end{array}$ & $\begin{array}{c}\text { Soma } \\
\text { quadrática } \\
\text { (SQ) }\end{array}$ & $\begin{array}{c}\text { Graus de } \\
\text { liberdade } \\
\text { (GL) }\end{array}$ & $\begin{array}{c}\text { Média } \\
\text { quadrática } \\
(\mathrm{MQ})\end{array}$ & $\mathrm{F}_{\text {calc }}$ & $p$ \\
\hline \multicolumn{6}{|c|}{ PCB 28} & \multicolumn{6}{|c|}{ PCB 52} \\
\hline Regressão & 513325.53 & 14 & 36666 & 5.4472 & 0.0107 & Regressão & 893877.91 & 14 & 63848 & 6.05694 & 0.0076 \\
\hline Resíduos & 53849.445 & 8 & 6731.2 & & & Resíduos & 84330.897 & 8 & 10541 & & \\
\hline F. ajuste & 28329.835 & 4 & 7082.5 & 1.11012 & 0.4609 & F. ajuste & 59470.905 & 4 & 14868 & 2.39223 & 0.2095 \\
\hline Erro puro & 25519.61 & 4 & 6379.9 & & & Erro puro & 24859.992 & 4 & 6215 & & \\
\hline Total & $5.67 \mathrm{E}+05$ & 22 & & & & Total & $1.73 \mathrm{E}+06$ & 22 & & & \\
\hline$\underline{\mathrm{R}^{2}}$ & 90.5057 & & & & & $\mathrm{R}^{2}$ & 91.379 & & & & \\
\hline \multicolumn{6}{|c|}{ PCB 74} & \multicolumn{6}{|c|}{ PCB 101} \\
\hline Regressão & 2840648.2 & 14 & 202903 & 6.89185 & 0.0079 & Regressão & 4428071.6 & 14 & 316291 & 9.58803 & 0.0016 \\
\hline Resíduos & 235528.7 & 8 & 29441 & & & Resíduos & 263904.81 & 8 & 32988 & & \\
\hline F. ajuste & 229643.8 & 4 & 57411 & 39.0225 & 0.0064 & F. ajuste & 258780.28 & 4 & 64695 & 50.4983 & 0.0011 \\
\hline Erro puro & 5884.9024 & 4 & 1471.2 & & & Erro puro & 5124.5352 & 4 & 1281.1 & & \\
\hline Total & $3.08 \mathrm{E}+06$ & 22 & & & & Total & 4.69E+06 & 22 & & & \\
\hline$\underline{\mathrm{R}^{2}}$ & 92.3435 & & & & & $\mathrm{R}^{2}$ & 94.3754 & & & & \\
\hline \multicolumn{6}{|c|}{ PCB 118} & \multicolumn{6}{|c|}{ PCB 153} \\
\hline$\overline{\text { Regressão }}$ & 11479535 & 14 & 819967 & 8.21801 & 0.0027 & Regressão & 100571003 & 14 & $7 \mathrm{E}+06$ & 4.33857 & 0.0216 \\
\hline Resíduos & 798214.76 & 8 & 99777 & & & Resíduos & 13246104 & 8 & $2 \mathrm{E}+06$ & & \\
\hline F. ajuste & 795570.51 & 4 & 198893 & 300.868 & $3 \mathrm{E}-05$ & F. ajuste & 13228883 & 4 & $3 E+06$ & 768.182 & $5 \mathrm{E}-06$ \\
\hline Erro puro & 2644.2526 & 4 & 661.06 & & & Erro puro & 17221.034 & 4 & 4305.3 & & \\
\hline Total & $1.23 \mathrm{E}+07$ & 22 & & & & Total & $1.14 \mathrm{E}+08$ & 22 & & & \\
\hline$\underline{\mathrm{R}^{2}}$ & 93.4987 & & & & & $\mathrm{R}^{2}$ & 88.3619 & & & & \\
\hline \multicolumn{6}{|c|}{ PCB 138} & \multicolumn{6}{|c|}{ PCB 187} \\
\hline Regressão & 12974491 & 14 & 926749 & 5.08859 & 0.0189 & Regressão & 31056671 & 14 & $2 \mathrm{E}+06$ & 4.95899 & 0.0143 \\
\hline Resíduos & 1456983.2 & 8 & 182123 & & & Resíduos & 3578689.1 & 8 & 447336 & & \\
\hline F. ajuste & 1454734.6 & 4 & 363684 & 646.954 & $1 \mathrm{E}-04$ & F. ajuste & 3577692.3 & 4 & 894423 & 3589.24 & $2 \mathrm{E}-07$ \\
\hline Erro puro & 2248.5906 & 4 & 562.15 & & & Erro puro & 996.78168 & 4 & 249.2 & & \\
\hline Total & $1.44 \mathrm{E}+07$ & 22 & & & & Total & $3.46 \mathrm{E}+07$ & 22 & & & \\
\hline$\underline{\mathrm{R}^{2}}$ & 89.9041 & & & & & $\mathrm{R}^{2}$ & & 89.6675 & & & \\
\hline \multicolumn{6}{|c|}{ PCB 128} & \multicolumn{6}{|c|}{ PCB 156} \\
\hline Regressão & 7982986.5 & 14 & 570213 & 6.22663 & 0.0069 & Regressão & 9340492.4 & 14 & 667178 & 4.83431 & 0.0155 \\
\hline Resíduos & 732612.93 & 8 & 91577 & & & Resíduos & 1104070.8 & 8 & 138009 & & \\
\hline F. ajuste & 732395.22 & 4 & 183099 & 3364.07 & 3E-07 & F. ajuste & 1103627.9 & 4 & 275907 & 2491.81 & 5E-07 \\
\hline Erro puro & 217.71092 & 4 & 54.428 & & & Erro puro & 442.90189 & 4 & 110.73 & & \\
\hline Total & $8.72 \mathrm{E}+06$ & 22 & & & & Total & $1.04 \mathrm{E}+07$ & 22 & & & \\
\hline$\underline{\mathrm{R}^{2}}$ & 91.5942 & & & & & $\mathrm{R}^{2}$ & 89.4292 & & & & \\
\hline \multicolumn{6}{|c|}{ PCB 180} & \multicolumn{6}{|c|}{ PCB 170} \\
\hline Regressão & 13363859 & 14 & 954561 & 4.5158 & 0.0191 & Regressão & 7582845.2 & 14 & 541632 & 3.27494 & 0.0484 \\
\hline Resíduos & 1691061 & 8 & 211383 & & & Resíduos & 1323094.8 & 8 & 165387 & & \\
\hline F. ajuste & 1689474.6 & 4 & 422369 & 1064.94 & 3E-06 & F. ajuste & 1322867.1 & 4 & 330717 & 5810.2 & 9E-08 \\
\hline Erro puro & 1586.4553 & 4 & 396.61 & & & Erro puro & 227.68009 & 4 & 56.92 & & \\
\hline Total & $1.51 \mathrm{E}+07$ & 22 & & & & Total & $8.91 \mathrm{E}+06$ & 22 & & & \\
\hline$\underline{\mathrm{R}^{2}}$ & 88.7674 & & & & & $\mathrm{R}^{2}$ & 85.1437 & & & & \\
\hline
\end{tabular}


Tabela 4. Comparação entre as melhores condições experimentais apresentadas pelo estudo univariado e pelo planejamento experimental

\begin{tabular}{|c|c|c|c|c|c|c|c|c|c|c|c|c|}
\hline \multicolumn{13}{|c|}{ Estudo univariado } \\
\hline PCB & 28 & 52 & 74 & 101 & 118 & 153 & 138 & 187 & 128 & 156 & 180 & 170 \\
\hline $\mathrm{C}_{\mathrm{NaCl}}(\% \mathrm{~m} / \mathrm{v})$ & 36 & 18 & 36 & 18 & 18 & 18 & 18 & 18 & 18 & 18 & 18 & 18 \\
\hline $\mathrm{T}\left({ }^{\circ} \mathrm{C}\right)$ & 80 & 80 & 80 & 80 & 80 & 80 & 80 & 80 & 80 & 80 & 80 & 80 \\
\hline $\mathrm{t}(\min )$ & 50 & 50 & 75 & 60 & 75 & 75 & 75 & 75 & 75 & 75 & 90 & 90 \\
\hline $\mathrm{C}_{\mathrm{MeOH}}(\% \mathrm{v} / \mathrm{v})$ & 5 & 5 & 5 & 5 & 5 & 5 & 5 & 5 & 5 & 5 & 5 & 5 \\
\hline \multicolumn{13}{|c|}{ Estudo multivariado } \\
\hline PCB & 28 & 52 & 74 & 101 & 118 & 153 & 138 & 187 & 128 & 156 & 180 & 170 \\
\hline $\mathrm{C}_{\mathrm{NaCl}}(\% \mathrm{~m} / \mathrm{v})$ & 36 & 36 & 36 & 36 & 36 & 36 & 36 & 36 & 27 & 27 & 27 & 27 \\
\hline $\mathrm{T}\left({ }^{\circ} \mathrm{C}\right)$ & 95 & 95 & 95 & 95 & 95 & 95 & 95 & 95 & 95 & 95 & 95 & 95 \\
\hline $\mathrm{t}(\min )$ & 70 & 100 & 62,5 & 62,5 & 62,5 & 70 & 70 & 70 & 70 & 70 & 70 & 70 \\
\hline $\mathrm{C}_{\mathrm{MeOH}}(\% \mathrm{v} / \mathrm{v})$ & 0 & 0 & 0 & 0 & 0 & 5 & 5 & 5 & 5 & 5 & 5 & 5 \\
\hline
\end{tabular}

\section{CONCLUSÕES}

O uso de planejamento experimental como ferramenta para otimizar a extração dos PCBs da matriz leite foi importante, pois identificou as melhores e piores condições experimentais para a extração desses analitos. Diferentes parâmetros de SPME ótimos foram obtidos para os diferentes PCBs analisados (tanto no estudo uni como multivariado), dificultando o ajuste de uma única condição para satisfazer o binômio eficiência de extração e uniformidade de extração para os 12 PCBs simultaneamente. No entanto, a análise das variâncias no estudo multivariado permitiu identificar que os parâmetros experimentais mais importantes para esse tipo de extração foram a temperatura e a força iônica do meio, sendo que o uso de altas temperaturas $\left(95^{\circ} \mathrm{C}\right)$ e a adição de $\mathrm{NaCl}(36 \% \mathrm{~m} / \mathrm{v})$ aumentaram a eficiência de extração para todos os PCBs estudados. Quando os demais parâmetros foram avaliados, observou-se um comportamento contrário, ou seja, melhores eficiências de extração foram obtidas com tempos de extração intermediários (70 min) e baixa concentração de $\mathrm{MeOH}$ no meio ( $5 \%$ v/v). O estudo permitiu verificar a contribuição de cada variável independentemente e a complexidade das interações que ocorrem entre elas, bem como a interdependência entre a eficiência de extração dos PCBs e os aspectos cinéticos e termodinâmicos da HS-SPME.

\section{MATERIAL SUPLEMENTAR}

As Figuras 1S a 4S encontram-se disponíveis, sob a forma de arquivo pdf, em http://quimicanova.sbq.org.br, com acesso livre.

\section{REFERÊNCIAS}

1. LeBlanc, G. A. Em A Textbook of modern toxicology; Hodgson, E.; Levi, P. E., eds.;. Appleton \& Lange: Samford, $2^{\text {nd }}$ ed., 1997, p. 389.

2. Jones, K. C.; Voogt, P. de; Environ. Pollut. 1999, 100, 209.

3. Heck, M. C.; Santos, J. S.; Bogusz Jr., S.; Costabeber, I.; Emanuelli, T.; Food Chem. 2007, 102, 288.

4. Johansen, P.; Muir, D.; Asmund, G.; Riget, F.; Sci. Total Environ. 2004, 31, 189 .

5. Schecter, A.; Cramer, P.; Boggess, K.; Stanley, J.; Olson, J. R.; Chemosphere 1997, 34, 1437.
6. Basheer, C.; Lee, H. K.; Obbard, J. P.; J. Chromatogr., A 2004, 1022, 161.

7. Kowalski, C. H.; Costa, J. G.; Godoy, H. T.; Augusto, F.; J. Braz. Chem. Soc. 2010, 21, 502.

8. Massart, D. L.; Vandeginste, B. G. M.; Buydens, L. M. C.; Jong, S. de; Lewi, P. J.; Smeyer-Verbeke, J.; Handbook of chemometrics and qualimetrics Part A, Elsevier: Amsterdam, 1997.

9. Araújo, P. W.; Brereton, R. G.; Trends Anal. Chem. 1996, 15, 63.

10. Lundstedt, T.; Seifert, E.; Abrano, L.; Thelin, B.; Nystron, A.; Pettersen, J.; Bergman, R.; Chemom. Intell. Lab. Syst. 1998, 42, 3.

11. Doehlert, D. H.; Appl. Statist. 1970, 19, 231.

12. Silva, G. A.; Tese de Doutorado, Universidade Estadual de Campinas, Brasil, 2007.

13. Ferreira, S. L. C.; Santos, W. N. L. dos; Quintella, C. M.; Barros Neto, B.; Bosque-Sendra, J. M.; Talanta 2004, 63, 1061.

14. Butine, W. L.; Weigend, A. S.; Complex Syst. 1991, 5, 603.

15. Teófilo, R. F.; Ferreira, M. M. C.; Quim. Nova 2006, 29, 338.

16. Caldas, L. F. S.; Francisco, B. B. A.; Pereira Netto, A. D.; Cassaella, R. J.; Microchem. J. 2011, 99, 118.

17. Lima, K. M. G.; Raimundo Jr., I. M.; Pimentel, M. F.; Sens. Actuators, B 2011, 160, 691 .

18. Fabrino, H. J. F.; Silveira, J. N.; Borges Neto, W.; Silva, J. B. B. da; Anal. Lett. 2010, 43, 508.

19. Aragão, N. M. de; Veloso, M. C. C.; Bispo, M. S.; de Andrade, J. B.; Quim. Nova 2009, 32, 2482.

20. Ferreira, S. L. C.; Jesus, R. M. de; Matos, G. D.; de Andrade, J. B.; Bruns, R. E.; Santos, W. N. L. dos; Cavalcante, D. D.; Vale, M. G. R.; Dessuy, M. B.; J. Braz. Chem. Soc. 2009, 20, 788.

21. Röhrig, L.; Meisch, H.-U.; Fresenius J. Anal. Chem. 2000, 366, 106.

22. Montes, R.; Ramil, M.; Rodriguez, I.; Rubí, E.; Cela, R.; J. Chromatogr., A 2006, 1124, 43.

23. Penalver, A.; Pocurrull, E.; Borrull, F.; Marcé, R. M.; Trends Anal. Chem. 1999, 18, 557.

24. Garcia-Falcon, M. S.; Cancho-Grande, B.; Simal-Gandara, J.; Water Res. 2004, 38, 1679.

25. Llompart, M.; Pazos, M.; Landín, P.; Cela, R.; Anal. Chem. 2001, 73, 5858. 